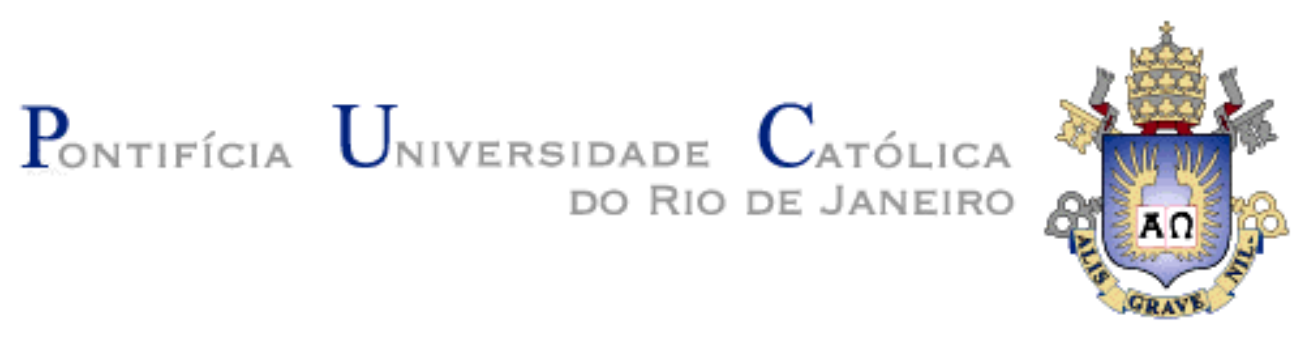

Kathy Camila Cardozo Osinski Senhorini

\title{
Guia Cilíndrico Corrugado com Dielétrico Anisotrópico
}

Tese de Doutorado

Tese apresentada ao Programa de Pós-graduação em Engenharia Elétrica da PUC-Rio como requisito parcial para obtenção do título de Doutor em Engenharia Elétrica.

Orientador: Prof. José Ricardo Bergmann Co-orientador: Prof. José Ricardo Descardeci 


\section{Kathy Camila Cardozo Osinski Senhorini}

\section{Guia Cilíndrico Corrugado com Dielétrico Anisotrópico}

Tese apresentada como requisito parcial para obtenção do grau de Doutor pelo Programa de Pós-Graduação em Engenharia Elétrica do Departamento de Engenharia Elétrica do Centro Técnico Científico da PUC-Rio. Aprovada pela Comissão Examinadora abaixo assinada.

Prof. José Ricardo Bergmann

Orientador

Centro de Estudos em Telecomunicações /PUC-Rio

Prof. José Ricardo Descardeci

Co-orientador

Universidade Federal do Tocantins /UFT

Prof. Flavio José Vieira Hasselmann

Centro de Estudos em Telecomunicações /PUC-Rio

Prof. Fernando José da Silva Moreira UFMG

Prof. João Crisostomo Weil Albuquerque Costa UFPA

Prof. Sandro Rogério Zang

Centro de Estudos em Telecomunicações /PUC-Rio

Prof. José Eugenio Leal

Coordenador Setorial do Centro

Técnico Científico - PUC-Rio

Rio de Janeiro, 13 de fevereiro de 2012 
Todos os direitos reservados. É proibida a reprodução total ou parcial do trabalho sem autorização da universidade, do autor e do orientador.

\section{Kathy Camila Cardozo Osinski Senhorini}

Graduou-se em engenharia elétrica (1985) pela EEL (Lins, SP), obteve o grau de mestre em engenharia elétrica (2004) pela EESC-USP. Desenvolveu suas atividades na área de sua formação profissional, sendo que desde 2004 é professora na UFT, onde ingressou no curso de ciência da computação, participou da comissão de criação dos cursos de engenharia elétrica e civil pelo REUNI na UFT, e a partir de 2009 passou a fazer parte do curso de engenharia elétrica, como professora e atualmente é coordenadora do mesmo.

Ficha Catalográfica

Senhorini, Kathy Camila Cardozo Osinski
Guia Cilíndrico Corrugado com Dielétrico
Anisotrópico / Kathy Camila Cardozo Osinski Senhorini;
orientador: José Ricardo Bergmann; co-orientador: José
Ricardo Descardeci - 2012.
155 f. il. (color.); $30 \mathrm{~cm}$
Tese (doutorado) - Pontifícia Universidade
Católica do Rio de Janeiro, Departamento de Engenharia
Elétrica, 2012.
Inclui bibliografia
1. Engenharia elétrica - Teses. 2. Antena. 3.
Corneta. 4. Alimentadores. 5. Guias de onda. I. Bergmann,
José Ricardo. II. Descardeci, José Ricardo III. Pontifícia
Universidade Católica do Rio de Janeiro. Departamento de
Engenharia Elétrica. VI. Título.


À minha família representada pelo meu esposo José Carlos Senhorini, meus filhos João Carlos e Thiago e minha mãe, Odália. 


\section{Agradecimentos}

Aos meus orientadores Bergmann e Descardeci que acreditaram em mim, por suas orientações, estímulos, amizade e principalmente, paciência.

Ao meu esposo José Carlos por sua dedicação, compreensão e companheirismo, mesmo nos momentos mais difíceis. Aos meus filhos João Carlos e Thiago pelo apoio. À minha mãe por seu incentivo.

Aos colegas do CETUC, pelo apoio e amizade, em especial ao André, Fábio, Fabrício, Juliana, Maiquel, Marco Aurélio, Pedro e Ramirez.

Aos professores do CETUC pelos ensinamentos e pela ajuda.

Aos funcionários da Vice-Reitoria para Assuntos Acadêmicos, em especial à Ana Lúcia e Célia pelo carinho e pela colaboração. À Alcina e demais funcionários do CETUC e da PUC-Rio.

A todos os amigos e familiares que de uma forma ou de outra me estimularam ou me ajudaram.

Ao CNPq e à PUC-Rio, pelos auxílios concedidos, sem os quais esse trabalho não poderia ter sido realizado. 


\section{Resumo}

Senhorini, Kathy Camila Cardozo Osinski; Bergmann, José Ricardo (Orientador); Descardeci, José Ricardo (Co-orientador). Guia Cilíndrico Corrugado com Dielétrico Anisotrópico. Rio de Janeiro, 2012. 155p. Tese de Doutorado - Departamento de Engenharia Elétrica, Pontifícia Universidade Católica do Rio de Janeiro.

Esta tese apresenta uma análise de uma estrutura de corneta cônica corrugada com dielétrico anisotrópico, com eixo óptico na direção axial da corneta. Esta configuração de corneta pode ser conseguida utilizando-se a estrutura de uma corneta corrugada com a adição de um cone dielétrico anisotrópico uniaxial. A anisotropia pode ser conseguida artificialmente dopandose o material dielétrico isotrópico ou utilizando-se uma técnica de perfuração na direção axial do cone. Esta técnica de perfuração é sugerida e apresentada nesta Tese. Partindo-se da geometria definida, foram desenvolvidas as expressões dos campos transversais e o equacionamento para a obtenção das curvas características da estrutura. É apresentado ainda um estudo comparativo dos campos transversais com os casos degenerados já conhecidos de guias cilíndricos, entre eles, guia cilíndrico corrugado oco, guia cilíndrico metálico com bastão dielétrico, guia cilíndrico corrugado com bastão dielétrico isotrópico. As expressões para o cálculo dos campos radiados distantes foram obtidas para o guia cilíndrico corrugado com dielétrico anisotrópico. Em adição, expressões para o cálculo dos campos radiados distantes para a corneta cilíndrica corrugada com dielétrico anisotrópico com pequenos ângulos de abertura (flare angle) utilizandose a aproximação por capa esférica foram obtidas e apresentadas. Estas expressões foram baseadas na terceira definição de Ludwig. Foram realizadas análises para estruturas já conhecidas geradas a partir da degeneração do caso mais geral apresentado nesta tese com o objetivo de validar a teoria desenvolvida e os resultados provaram a validade do material teórico desenvolvido. Finalmente, foi desenvolvida uma análise paramétrica da estrutura apresentada com o intuito de verificar o comportamento dos campos radiados e da polarização cruzada máxima em função da anisotropia e da frequência de operação. Verificou-se que a largura de feixe de meia potência foi pouco influenciada pela anisotropia do dielétrico e apresentou comportamento esperado em função da frequencia. A variação da máxima polarização cruzada em função 
da frequência foi estudada. Em determinados valores de permissividade e anisotropia, para a configuração considerada, foi alcançada a condição híbrida balanceada. Nesta condição a estrutura apresentou baixos níveis de máxima polarização cruzada em uma faixa larga de frequência. Comportamento este muito superior ao da corneta corrugada, corneta metálica com dielétrico e corneta corrugada com dielétrico isotrópico.

\section{Palavras-chave}

Antena; Cornetas; Alimentadores; Guias de Onda. 


\section{Abstract}

Senhorini, Kathy Camila Cardozo Osinski; Bergmann, José Ricardo (Advisor); Descardeci, José Ricardo (Co-advisor). Anisotropic Dielectric Corrugated Cylindrical Guide. Rio de Janeiro, 2012. 155p. Doctoral Thesis - Departamento de Engenharia Elétrica, Pontifícia Universidade Católica do Rio de Janeiro.

This thesis presents an analysis of a corrugated conic horn structure with anisotropic dielectric. This dielectric cone is positioned inside the horn and has an optical axis in its longitudinal direction. This horn configuration can be obtained using the structure of corrugated horn and adding an anisotropic dielectric cone. The anisotropy can artificially be obtained by doping the isotropic dielectric or using a perforation technique. This perforation technique is suggested and presented in this Thesis. Expressions for the transverse fields and the propagation constant curves were developed considering this new geometry. It is also presented a comparative study of the degenerated transversal fields with well-known structures. These structures are: hollow cylindrical guide, corrugated cylindrical guide, cylindrical metallic guide with dielectric and corrugated cylindrical guide with dielectric. The expressions to calculate the radiated far fields were obtained for the corrugated cylindrical guide with anisotropic dielectric. In addition, expressions to calculate the radiated far fields for the corrugated cylindrical horn with anisotropic dielectric were developed and presented. These expressions considered horns with small flare angle and used the spherical cap approximation theory. All expressions considered the 3th Ludwig definition for the radiated far field. Far field analyses were conducted for well-known structures. These structures were obtained by degenerating the new configuration presented in this thesis and aimed to validate the theoretical developed theory. The results proved its validity. Finally, a parametric analysis was performed. This analysis considered, for a given structure configuration, the behavior of the radiated far fields and its maximum cross polar levels as a function of the anisotropy and frequency. It was verified that the dielectric anisotropy had very little effect on the half power beam width characteristic. The maximum cross polar level as a function of the frequency was analyzed. For some specific values of permittivity and anisotropy, for the considered 
configuration, the balanced hybrid condition was reached. In this condition the structure presented very low cross polar values in a large frequency band. This behavior is much more superior to the corrugated horn, dielectric horn and the isotropic dielectric corrugated horn.

\section{Keywords}

Antenna; Horns; Antenna Feeds; Waveguides. 


\section{Sumário}

1 Introdução

2 Desenvolvimento das Expressões dos Campos no Interior do Guia Cilíndrico Corrugado com Bastão Dielétrico Anisotrópico 24

2.1. Introdução 24

2.2. Geometria da Estrutura Analisada $\quad 24$

2.3. Expressões dos Campos 26

2.4. Estudo Teórico da Equação Característica para Casos Degenerados 35

2.4.1. Guia Cilíndrico Corrugado Oco 36

2.4.2. Guia Metálico Liso com Bastão Dielétrico 40

2.4.3. Guia Cilíndrico Corrugado com Bastão Dielétrico Isotrópico 43

2.4.4. Guia Metálico Cilíndrico Corrugado com Bastão Dielétrico Anisotrópico 46

3 Expressões para os Campos Eletromagnéticos 49

3.1. Campos na Abertura 49

3.1.1. Região Dielétrica Anisotrópica $\left(r \leq r_{1}\right) \quad 49$

3.1.2. Região do Guia fora do Dielétrico $\left(r_{1} \leq r \leq r_{0}\right)$

3.2. Campo Radiado Distante 55

3.2.1. Campo Radiado devido à Região i 56

3.3. Campo Radiado devido à Região o 61

3.3.1. Campo Radiado devido à Abertura Completa 64

3.4. Campo Radiado Distante para Corneta (Aproximação por fase esférica) 66

3.4.1. Introdução 66

3.4.2. Corneta Cônica Corrugada com Núcleo Dielétrico Anisotrópico 67

3.5. Caso Particular: Simulação do Guia Anisotrópico com Anisotropia criada a partir da Técnica de Perfuração do Bastão Dielétrico 69

4 Análise do Comportamento dos Campos Transversais na Estrutura
Guiada em Estudo

4.1. Introdução 72

4.2. Estruturas Conhecidas 72

4.2.1. Guia Cilíndrico Oco (modo principal TE11) 72

4.2.2. Guia Cilíndrico Corrugado (modos Híbridos) 73 
4.2.2.1. Modo EH11 74

4.2.2.2. Modo HE11 75

4.2.3. Guia Cilíndrico Metálico Liso com Bastão Dielétrico (modos Híbridos) 75

4.2.3.1. Modo $\mathrm{EH} 11$

4.2.3.2. Modo HE11 77

4.2.4. Guia Cilíndrico Corrugado com Bastão Dielétrico Isotrópico

(modos Híbridos) 77

4.2.4.1. Modo EH11 78

4.2.4.2. Modo HE11 79

4.3. Estrutura em estudo: Guia Cilíndrico Corrugado com Bastão

Dielétrico Anisotrópico $\quad 80$

4.3.1. Efeito da Anisotropia no Bastão Dielétrico nos Campos Transversais 80

4.3.1.1. Dielétrico Isotrópico, $\varepsilon_{\mathrm{r}}=\varepsilon_{\mathrm{z}}=\varepsilon_{\mathrm{t}}=1,5 \quad 80$

4.3.1.2. Dielétrico Anisotrópico, $\varepsilon_{\mathrm{z}}=1,5, \varepsilon_{\mathrm{t}}=1,1$

4.3.1.3. Dielétrico Anisotrópico, $\varepsilon_{\mathrm{z}}=1,1, \varepsilon_{\mathrm{t}}=1,5$

4.4. Discussão e Conclusões 84

5 Análise Paramétrica $\quad 85$

5.1. Análise Paramétrica para o Guia Cilíndrico Corrugado com

Dielétrico Anisotrópico 85

5.2. Efeito da Anisotropia do Dielétrico sobre a Freqüência de Corte 86

5.2.1.1. Variação da Permissividade Longitudinal 86

5.2.1.2. Variação da Permissividade Transversal 88

5.2.2. Influência da Permissividade do Dielétrico sobre o Campo Radiado 91

5.2.3. Influência da Freqüência nos Padrões de Radiação e Polarização

Cruzada 99

5.3. Análise Paramétrica para a Corneta com ângulo flare de 12 Graus 106

5.3.1. Efeito do ângulo de flare no Diagrama de Radiação 106

5.3.2. Influência da Freqüência nos Padrões de Radiação e Polarização

Cruzada 109

5.4 Discussões e Conclusões 113

6 Conclusões 115

6.1. Introdução 115

6.2. Trabalhos Futuros 120

6.3. Dificuldades Encontradas 120

6.4. Contribuição da Pesquisa 121 
Referências Bibliográficas

APENDICE A Obtenção dos Potenciais Vetores elétrico e magnético de Hertz [9].

A.1 Potencial Vetor de Hertz do Tipo Elétrico 125

A.2 Potencial Vetor de Hertz do Tipo Magnético 126

A.3 Solução Geral com os Potenciais Vetores de Hertz dos Tipos

Elétrico e Magnético

APÊNDICE B Determinação dos Campos Elétrico e Magnético a partir dos Potenciais Vetores de Hertz

APENDICE C Obtenção da Admitância de Superfície em $r=r_{0}$ no Lado da Corrugação.

APENDICE D Obtenção dos Coeficientes $A_{n}, B_{n}, C_{n}, D_{n}, E_{n}, F_{n}$

APÊNDICE E Desenvolvimento de Integrais de Campos usadas [10,14]

E. $1 \operatorname{Cos}(n \phi) \operatorname{sen}\left(\phi-\phi^{\prime}\right)$

E. $2 \operatorname{Cos}(n \phi) \cos \left(\phi-\phi^{\prime}\right)$

148

E.3 Sen $\left(n \phi^{\prime}\right) \operatorname{sen}\left(\phi-\phi^{\prime}\right)$

149

E. $4 \operatorname{Sen}(n \phi) \cos \left(\phi-\phi^{\prime}\right)$

151

E.5 Obtenção das Integrais para o Caso Especial em que $n=1$

152

E.6 Desenvolvimento para os Casos Apresentados 


\section{Lista de figuras}

Figura 1.1 - Cornetas mono-modo [4]. 19

Figura 1.2 - Cornetas Multímodo. $\quad 20$

Figura 1.3 - Tipos de alimentadores corrugados [5]: a) guia cilíndrico corrugado, b) corneta corrugada com ângulo flare estreito, c) corneta $\begin{array}{ll}\text { corrugada profile. } & 20\end{array}$

Figura 1.4 - Corneta cônica com carregamento dielétrico [4]. 21

Figura 2.1 - Vista do guia cilíndrico corrugado com núcleo dielétrico. 25

Figura 2.2 - Relação entre áreas transversais do furo e do bastão dielétrico. 26

Figura 2.3 - Polarização Cruzada Máxima como função da

freqüência normalizada,onde f0 é a freqüência para mínima polarização

cruzada. Parâmetro: diâmetro de abertura normalizado [5].

Figura 2.4 - Curvas características do guia cilíndrico corrugado oco

para os modos com $\mathrm{n}=0,1$ e 2 e $\mathrm{m}=1,2$, com $\mathrm{r}_{0}=63,17 \mathrm{~mm}$,

$\mathrm{r}_{2}=71,17 \mathrm{~mm}, \mathrm{~d}=14 \mathrm{~mm}$.

Figura 2. 5 - Curvas de Dispersão Simuladas para uma configuração

conhecida com resultados já apresentados na literatura [4], onde

$r_{1}=50,54 \mathrm{~mm}, r_{0}=63,17 \mathrm{~mm}$ e profundidade de corrugação

$\mathrm{d}=14 \mathrm{~mm}$. Núcleo dielétrico isotrópico com $\varepsilon_{\mathrm{r}}=1,05$.

Figura 2.6 - Curvas de dispersão dos n modos para o guia cilíndrico de parede lisa com bastão dielétrico isotrópico, conforme Figura 2.1, sem as corrugações, $\varepsilon_{r}=1,15, r_{0}=63,17 \mathrm{~mm}, r_{1}=0,8 r_{0}$.

Figura 2.7 - Curvas de Dispersão Simuladas para uma configuração de guia cilíndrico corrugado com núcleo dielétrico isotrópico com $\varepsilon_{r}=3,745$, $r_{1}=50,54 \mathrm{~mm}, r_{0}=63,17 \mathrm{~mm}$ e profundidade de corrugação $\mathrm{d}=8 \mathrm{~mm}$.

Figura 2.8 - Curvas de Dispersão Simuladas para uma configuração de guia cilíndrico corrugado com núcleo dielétrico isotrópico para dois casos, um com $\varepsilon_{r}=1,05$ e outro com $\varepsilon_{r}=3,745$; parâmetros $r_{1}=50,54 \mathrm{~mm}$, $r_{0}=63,17 \mathrm{~mm}$ e profundidade de corrugação $d=8 \mathrm{~mm}$.

Figura 2.9 - Curvas de dispersão para os dois primeiros modos para a configuração do guia cilíndrico corrugado com dielétrico anisotrópico apresentada na Figura 2.1, para os casos onde $\varepsilon_{\mathrm{t}}=1,5$ e $\varepsilon_{\mathrm{z}}=1,3$ e para $\varepsilon_{\mathrm{r}}=1,5$. Parâmetros: $r_{0}=63,17 \mathrm{~mm}, \mathrm{r}_{1}=50,54 \mathrm{~mm}$, $r_{2}=r_{0}+d$, com $d=14 \mathrm{~mm}$. 
Figura 3.1 - Vista lateral de uma corneta cônica corrugada, onde $r_{0}$ varia com o ângulo de abertura, $\theta$ e $\mathrm{R}=\mathrm{r}_{0} / \operatorname{sen} \theta$.

Figura 3.2 - Curvas de dispersão simuladas. Guia cilíndrico corrugado com dielétrico isotrópico perfurado para simular anisotropia: $\varepsilon_{r}=10,3$ (alumina ceramica), com $r_{1}=50,54 \mathrm{~mm}, r_{0}=63,17 \mathrm{~mm}$ e profundidade de corrugação $d=8 \mathrm{~mm}$. Anisotropia criada inserindo 450 furos axiais, com diametro $\phi=4 \mathrm{~mm}$, no dielétrico, resultando em $\varepsilon_{\mathrm{t}}=2,745$ e $\varepsilon_{z}=3,745$. Dielétrico isotrópico $\varepsilon_{r}=3,745$.

Figura 3.3 - Padrões de radiação para o guia cilíndrico corrugado com dielétrico isotrópico e com dielétrico anisotrópico, com $r_{1}=50,54 \mathrm{~mm}$, $r_{0}=63,17 \mathrm{~mm}$ e profundidade de corrugação $\mathrm{d}=8 \mathrm{~mm}$. Anisotropia criada inserindo 450 furos axiais, com diametro $\phi=4 \mathrm{~mm}$, no dielétrico isotrópico com permissividade $\varepsilon_{\mathrm{r}}=10,3$, resultando em anisotropia com $\varepsilon_{t}=2,745$ e $\varepsilon_{z}=3,745$. Dielétrico isotrópico $\varepsilon_{r}=3,745$.

Figura 4.1 - Campo Vetorial na abertura circular do modo TE11 no plano terra, para guia cilíndrico oco, $a=r_{0}=63,17 \mathrm{~mm}$.

Figura 4.2 - Guia cilíndrico corrugado com raio interno $r_{0}=63,17 \mathrm{~mm}$ e profundidade de corrugação $d=14 \mathrm{~mm}$, com $r_{2}=r_{0}+d$.

Figura 4.3 - Campo Vetorial na abertura circular do guia corrugado do modo $\mathrm{EH} 11$, com $\mathrm{r}_{0}=63,17 \mathrm{~mm}, \mathrm{~d}=14 \mathrm{~mm}, \mathrm{r}_{2}=77,17 \mathrm{~mm}, \mathrm{k}_{0}=48,05$, $\beta / k_{0}=0,941$.

Figura 4.4 - Campo Vetorial na abertura circular do guia corrugado para o modo $H E 11$, parâmetros $r_{0}=63,17 \mathrm{~mm}, \mathrm{~d}=14 \mathrm{~mm}, \mathrm{r}_{2}=77,17 \mathrm{~mm}$ $\mathrm{k}_{0}=112,28, \beta / \mathrm{k}_{0}=0,941$.

Figura 4.5 - Campo Vetorial na abertura circular do guia cilíndrico metálico com dielétrico de $\varepsilon_{r}=1,15$, do modo $E H 11, r_{0}=63,17 \mathrm{~mm}, \mathrm{r} 1=50,54 \mathrm{~mm}$, $\mathrm{k}_{0}=200, \beta / \mathrm{k}_{0}=1,4$.

Figura 4.6 - Campo Vetorial na abertura circular para o guia cilíndrico metálico com dielétrico de $\varepsilon_{\mathrm{r}}=1,15$ do modo $\mathrm{HE} 11$, raio $\mathrm{r}_{0}=63,17 \mathrm{~mm}$, $\mathrm{r} 1=50,54 \mathrm{~mm}, \mathrm{k}_{0}=200, \beta / \mathrm{k}_{0}=0,9756$.

Figura 4.7 - Campo Elétrico na Abertura do guia cilíndrico corrugado com bastão dielétrico, $\mathrm{EH} 11, \varepsilon_{\mathrm{z}}=\varepsilon_{\mathrm{t}}=1,15, \mathrm{r}_{0}=63,17 \mathrm{~mm}, \mathrm{r}_{1}=50,54 \mathrm{~mm}$, $\mathrm{r}_{2}=77,17 \mathrm{~mm}, \mathrm{k}_{0}=51,25, \beta / \mathrm{k}_{0}=1,0544$.

Figura 4.8 - Campo Elétrico na Abertura do guia cilíndrico corrugado com bastão dielétrico isotrópico, modo $\mathrm{HE} 11, \varepsilon_{\mathrm{z}}=\varepsilon_{\mathrm{t}}=1,15, \mathrm{r}_{0}=63,17 \mathrm{~mm}$, $r_{1}=50,54 \mathrm{~mm}, r_{2}=77,17 \mathrm{~mm}, \mathrm{k}_{0}=200, \beta / \mathrm{k}_{0}=1,0544$. 
Figura 4.9 - Campo elétrico para modo HE11 de um guia cilíndrico

corrugado com dielétrico isotrópico com permissividade relativa igual a 1,5.

a) $\mathrm{k}_{0}=65, \beta / \mathrm{k}_{0}=1,0177 ;$ b) $\mathrm{k} 0=85, \beta / \mathrm{k}_{0}=1,1233$; c) $\mathrm{k}_{0}=105, \beta / \mathrm{k}_{0}=1,1607$;

d) $\mathrm{k}_{0}=150, \beta / \mathrm{k}_{0}=1,1927$.

Figura 4.10 - Campo elétrico para modo HE11 de um guia cilíndrico corrugado com dielétrico anisotrópico com permissividade $\varepsilon_{z}=1,5, \varepsilon_{t}=1,1$.

a) $k_{0}=65, \beta / k_{0}=1,013$; b) $k_{0}=85, \beta / k_{0}=1,1273$; c) $k_{0}=105, \beta / k_{0}=1,1636$;

d) $k_{0}=150, \beta / k_{0}=1,1940$.

Figura 4.11 - Campo elétrico para modo HE11 de um guia cilíndrico corrugado com dielétrico anisotrópico com $\varepsilon_{\mathrm{z}}=1,1$ e $\varepsilon_{\mathrm{t}}=1,5$. a) $\mathrm{k}_{0}=65$, $\left.\beta / k_{0}=0,81092 ; b\right) k_{0}=85, \beta / k_{0}=0,9322$; c) $k_{0}=105, \beta / k_{0}=0,97726$;

d) $k_{0}=150, \beta / k_{0}=1,0152$.

Figura 5.1 - Curvas de dispersão do modo EH11, para o guia cilíndrico corrugado com dielétrico anisotrópico definido no Capítulo 2 , mantendo $\varepsilon_{\mathrm{t}}$ constante e igual a 1,5 e variando $\varepsilon_{\mathrm{z}}\left(\mathrm{r}_{0}=63,17 \mathrm{~mm}, \mathrm{r}_{1}=0,8 \mathrm{r}_{0}\right.$, $\left.r_{2}=r_{0}+d, d=14 \mathrm{~mm}\right)$.

Figura 5.2 - Curvas de dispersão do modo HE11 para o guia cilíndrico corrugado com dielétrico anisotrópico definido no Capítulo 2, mantendo $\varepsilon_{\mathrm{t}}$ constante e igual a 1,5 e variando $\varepsilon_{\mathrm{z}}\left(r_{0}=63,17 \mathrm{~mm}, \mathrm{r}_{1}=0,8 \mathrm{r}_{0}, \mathrm{r}_{2}=\mathrm{r}_{0}+\mathrm{d}\right.$, $\mathrm{d}=14 \mathrm{~mm})$.

Figura 5.3 - Curvas de dispersão do modo EH11 para o guia cilíndrico corrugado com dielétrico anisotrópico definido no Capítulo 2, mantendo $\varepsilon_{\mathrm{z}}=1,5$ constante e variando $\varepsilon_{\mathrm{t}}\left(\mathrm{r}_{0}=63,17 \mathrm{~mm}, \mathrm{r}_{1}=0,8 \mathrm{r}_{0}, \mathrm{r}_{2}=\mathrm{r}_{0}+\mathrm{d}\right.$, $\mathrm{d}=14 \mathrm{~mm})$.

Figura 5.4 - Curvas de dispersão do modo HE11 para o guia cilíndrico corrugado com dielétrico anisotrópico definido no Capítulo 2, mantendo $\varepsilon_{\mathrm{z}}=1,5$ constante e variando $\varepsilon_{\mathrm{t}}\left(r_{0}=63,17 \mathrm{~mm}, \mathrm{r}_{1}=0,8 \mathrm{r}_{0}, \mathrm{r}_{2}=\mathrm{r}_{0}+\mathrm{d}\right.$, $\mathrm{d}=14 \mathrm{~mm})$.

Figura 5.5 - Curvas demonstrando o comportamento da freqüência de corte inferior em relação à anisotropia para o guia cilíndrico corrugado com núcleo dielétrico anisotrópico em estudo definido no Capítulo 2 $\left(r_{0}=63,17 \mathrm{~mm}, r_{1}=0,8 r_{0}, r_{2}=r_{0}+d, d=14 \mathrm{~mm}\right)$.

Figura 5.6 - Diagramas de radiação do modo HE11, das polarizações principal e cruzada, no plano $\phi=45^{\circ}$, para o guia cilíndrico corrugado com dielétrico anisotrópico, de um caso degenerado com $\varepsilon_{\mathrm{r}}=\varepsilon_{\mathrm{z}}=\varepsilon_{\mathrm{t}}=1,05$ e para vários casos anisotrópicos mantendo $\varepsilon_{\mathrm{t}}=1,5 \mathrm{e}$ variando $\varepsilon_{\mathrm{z}}$. 
Parâmetros: $r_{0}=63,17 \mathrm{~mm}, r_{1}=0,8 r_{0}, r_{2}=r_{0}+d, d=14 \mathrm{~mm}, f=5,36 \mathrm{GHz}$.

Figura 5.7 - Distribuição da intensidade do campo elétrico transversal na abertura do guia cilíndrico corrugado com dielétrico anisotrópico do modo $\mathrm{HE} 11$, de vários casos anisotrópicos mantendo $\varepsilon_{z}=1,5 \mathrm{e}$ variando $\varepsilon_{\text {t. }}$. Cortes nos planos $Y Z$ e XZ. Parâmetros: $r_{0}=63,17 \mathrm{~mm}$, $r_{1}=0,8 r_{0}, r_{2}=r_{0}+d, d=14 \mathrm{~mm}, f=5,36 \mathrm{GHz}$

Figura 5.8 - Comparação entre distribuição da intensidade do campo elétrico transversal normalizado na abertura do guia cilíndrico corrugado com dielétrico anisotrópico do modo HE11, dos casos anisotrópicos $\varepsilon_{\mathrm{t}}=1.5$ e $\varepsilon_{\mathrm{z}}=1,1 ; \varepsilon_{\mathrm{t}}=1,5$ e $\varepsilon_{\mathrm{z}}=1,9$. Cortes nos planos $\mathrm{YZ}$ e $X Z$.

Parâmetros: $r_{0}=63,17 \mathrm{~mm}, r_{1}=0,8 r_{0}, r_{2}=r_{0}+d, d=14 \mathrm{~mm}, f=5,36 \mathrm{GHz}$.

Figura 5.9 - Diagramas de radiação do modo HE11, para as polarizações principal e cruzada, no plano $\phi=45^{\circ}$, para o guia cilíndrico corrugado com dielétrico anisotrópico, de um caso degenerado com $\varepsilon_{\mathrm{r}}=\varepsilon_{\mathrm{z}}=\varepsilon_{\mathrm{t}}=1,05$ (corrugado oco), e de vários casos anisotrópicos mantendo $\varepsilon_{\mathrm{z}}=1,5$ e variando $\varepsilon_{\mathrm{t}}$. Parâmetros: $\mathrm{r}_{0}=63,17 \mathrm{~mm}$, $r_{1}=0,8 r_{0}, r_{2}=r_{0}+d, d=14 \mathrm{~mm}, f=5,36 \mathrm{GHz}$.

Figura 5.10 - Distribuição da intensidade do campo elétrico transversal na abertura do guia cilíndrico corrugado com dielétrico anisotrópico do modo $\mathrm{HE} 11$, de vários casos anisotrópicos mantendo $\varepsilon_{\mathrm{t}}=1,5 \mathrm{e}$ variando $\varepsilon_{z}$. Cortes nos planos $Y Z$ e XZ. Parâmetros: $r_{0}=63,17 \mathrm{~mm}$, $r_{1}=0,8 r_{0}, r_{2}=r_{0}+d, d=14 \mathrm{~mm}, f=5,36 \mathrm{GHz}$.

Figura 5.11 - Comparação entre distribuição da intensidade do campo elétrico transversal normalizado na abertura do guia cilíndrico corrugado com dielétrico anisotrópico do modo HE11, dos casos anisotrópicos $\varepsilon_{\mathrm{z}}=1,5$ e $\varepsilon_{\mathrm{t}}=1.1 ; \varepsilon_{\mathrm{z}}=1,5$ e $\varepsilon_{\mathrm{t}}=1$,9. Cortes nos planos $\mathrm{YZ}$ e $\mathrm{XZ}$.

Parâmetros: $r_{0}=63,17 \mathrm{~mm}, r_{1}=0,8 r_{0}, r_{2}=r_{0}+d, d=14 \mathrm{~mm}, f=5,36 \mathrm{GHz}$.

Figura 5.12 - Largura de Feixe de Meia Potência (-3 dB) em função da permissividade para o guia cilíndrico corrugado com dielétrico anisotrópico mantendo $\varepsilon_{\mathrm{t}}=1,5 \mathrm{e}$ variando $\varepsilon_{\mathrm{z}} \mathrm{e}$, mantendo $\varepsilon_{\mathrm{z}}=1,5 \mathrm{e}$ variando $\varepsilon_{\text {t. }}$ Parâmetros: $r_{0}=63,17 \mathrm{~mm}, r_{1}=0,8 r_{0}, r_{2}=r_{0}+d, d=14 \mathrm{~mm}$, $f=5,36 \mathrm{GHz}$.

Figura 5.13 - Nível de Polarização Cruzada Máxima em função da permissividade para o guia cilíndrico corrugado com dielétrico anisotrópico em estudo, mantendo $\varepsilon_{\mathrm{t}}=1,5$ e variando $\varepsilon_{\mathrm{z}} \mathrm{e}$, mantendo $\varepsilon_{\mathrm{z}}=1,5$ e variando $\varepsilon_{\mathrm{t}}$. Parâmetros: $\mathrm{r}_{0}=63,17 \mathrm{~mm}, \mathrm{r}_{1}=0,8 \mathrm{r}_{0}$, 
$r_{2}=r_{0}+d, d=14 \mathrm{~mm}, f=5,36 \mathrm{GHz}$

Figura 5.14 - Gráfico do Nível da Largura de Feixe de -3 dB em função da freqüência para o guia cilíndrico corrugado com dielétrico anisotrópico em estudo, mantendo $\varepsilon_{t}=1,5$ constante e variando $\varepsilon_{z}$. Parâmetros: $r_{0}=63,17 \mathrm{~mm}, r_{1}=0,8 r_{0}, r_{2}=r_{0}+d, d=14 \mathrm{~mm}$.

Figura 5.15 - Gráfico do Nível da Largura de Feixe de $-3 \mathrm{~dB}$ em função da freqüência para o guia cilíndrico corrugado com dielétrico anisotrópico em estudo, mantendo $\varepsilon_{\mathrm{z}}=1,5$ constante e variando $\varepsilon_{\mathrm{t}}$. Parâmetros: $r_{0}=63,17 \mathrm{~mm}, r_{1}=0,8 r_{0}, r_{2}=r_{0}+d, d=14 \mathrm{~mm}$.

Figura 5.16 - Nível de Máxima Polarização Cruzada em função da freqüência de operação para o guia cilíndrico corrugado oco, guia cilíndrico metálico com bastão dielétrico (sem corrugação) $\operatorname{com} \varepsilon_{\mathrm{r}}=1,5$ e guia cilíndrico corrugado com bastão dielétrico isotrópico com $\varepsilon_{\mathrm{r}}=1,5$. Parâmetros: $r_{0}=63,17 \mathrm{~mm}, r_{1}=0,8 r_{0}, r_{2}=r_{0}+d, d=14 \mathrm{~mm}$.

Figura 5.17 - Gráfico do Nível da Polarização Cruzada Máxima em função da freqüência de operação para o guia cilíndrico corrugado com dielétrico anisotrópico, de um caso degenerado com $\varepsilon_{\mathrm{r}}=\varepsilon_{\mathrm{z}}=\varepsilon_{\mathrm{t}}=1$ e de vários casos anisotrópicos mantendo $\varepsilon_{\mathrm{z}}=1,5$ e variando $\varepsilon_{\mathrm{t}}$. Parâmetros: $r_{0}=63,17 \mathrm{~mm}, r_{1}=0,8 r_{0}, r_{2}=r_{0}+d, d=14 \mathrm{~mm}$.

Figura 5.18 - Gráfico do Nível de Polarização Cruzada Máxima em função da freqüência de operação para o guia cilíndrico corrugado com dielétrico anisotrópico mantendo $\varepsilon_{\mathrm{t}}=1,5$ e variando $\varepsilon_{\mathrm{z}}\left(\varepsilon_{\mathrm{t}}=1,5, \varepsilon_{\mathrm{z}}\right.$ variando de 1,1 a 1,9). Parâmetros: $r_{0}=63,17 \mathrm{~mm}, r_{1}=0,8 r_{0}, r_{2}=r_{0}+d, d=14 \mathrm{~mm}$. Figura 5.19 - Gráfico do Nível de Polarização Cruzada Máxima em função da freqüência de operação para o guia cilíndrico corrugado oco $\left(\varepsilon_{r}=1,05\right)$, guia cilíndrico metálico dielétrico ( $\varepsilon_{r}=1,5$ sem corrugações), guia cilíndrico corrugado com dielétrico: isotrópico com $\varepsilon_{\mathrm{r}}=1,5$, anisotrópico com $\varepsilon_{\mathrm{z}}=1,5$ e $\varepsilon_{\mathrm{t}}=1,3$ e anisotrópico com $\varepsilon_{\mathrm{z}}=1,9$ e $\varepsilon_{\mathrm{t}}=1,5$.

Parâmetros: $r_{0}=63,17 \mathrm{~mm}, r_{1}=0,8 r_{0}, r_{2}=r_{0}+d, d=14 \mathrm{~mm}$.

Figura 5.20 - Diagramas de radiação para corneta cônica corrugada com dielétrico anisotrópico, para freqüência de $5,36 \mathrm{GHz}$, ângulo flare de 12․ Parâmetros: $r_{0}=63,17 \mathrm{~mm}, r_{1}=0,8 r_{0}, r_{2}=r_{0}+d, d=14 \mathrm{~mm}$. Figura 5.21 - Diagramas de radiação para casos degenerados de guia cilíndrico corrugado com dielétrico anisotrópico e para corneta cônica corrugada com dielétrico anisotrópico, para freqüência de $5,36 \mathrm{GHz}$, ângulo 
flare de 12․ Parâmetros: $r_{0}=63,17 \mathrm{~mm}, r_{1}=0,8 r_{0}, r_{2}=r_{0}+d, d=14 \mathrm{~mm} . \quad 108$ Figura 5.22 - Diagramas de radiação para casos degenerados de guia cilíndrico corrugado com dielétrico anisotrópico e para corneta cônica corrugada com dielétrico anisotrópico, para freqüência de $5,36 \mathrm{GHz}$, ângulo flare de $12^{\circ}$. Parâmetros: $r_{0}=63,17 \mathrm{~mm}, r_{1}=0,8 r_{0}, r_{2}=r_{0}+d$, $\mathrm{d}=14 \mathrm{~mm}$.

Figura 5.23 - Diagramas de radiação em função da freqüência para corneta cônica corrugada com núcleo dielétrico isotrópico com dimensões: $r_{0}=63,17 \mathrm{~mm}, r_{1}=0,8 r_{0}, r_{2}=r_{0}+d, d=14 \mathrm{~mm}$, ângulo flare $=12^{\circ}$. Casos:

a) $\varepsilon_{\mathrm{r}}=1,05$; b) $\varepsilon_{\mathrm{r}}=1,5$.

Figura 5.24 - Diagramas de radiação em função da freqüência para corneta cônica corrugada com núcleo dielétrico anisotrópico com dimensões: $r_{0}=63,17 \mathrm{~mm}, r_{1}=0,8 r_{0}, r_{2}=r_{0}+d, d=14 \mathrm{~mm}$, ângulo flare $=12^{\circ}$. Casos:

a) $\varepsilon_{\mathrm{z}}=1,5$ e $\varepsilon_{\mathrm{t}}=1,1$, b) $\varepsilon_{\mathrm{z}}=1,5$ e $\varepsilon_{\mathrm{t}}=1,3$, c) $\varepsilon_{\mathrm{z}}=1,5$ e $\varepsilon_{\mathrm{t}}=1,7$, d) $\varepsilon_{\mathrm{z}}=1,5$ e $\varepsilon_{t}=1,9$.

Figura 5.25 - Nível de Largura de Feixe de -3 dB para guia cilíndrico corrugado com dielétrico anisotrópico e para corneta cônica corrugada com dielétrico anisotrópico e ângulo "flare" de $12^{\circ}$ em função da permissividade, mantendo $\varepsilon_{\mathrm{z}}=1,5$ e variando $\varepsilon_{\mathrm{t}}$, para a freqüência de 5,36 GHz. Parâmetros: $r_{0}=63,17 \mathrm{~mm}, r_{1}=0,8 r_{0}, r_{2}=r_{0}+d, d=14 \mathrm{~mm}$.

Figura 5.26 - Máxima polarização cruzada em função da freqüência para corneta cônica corrugada com núcleo dielétrico anisotrópico, ângulo "flare" de $12^{\circ}$ e guia cilíndrico corrugado com dielétrico anisotrópico mantendo $\varepsilon_{\mathrm{z}}=1,5$ e variando $\varepsilon_{\mathrm{t}}$ e para os casos isotrópico com $\varepsilon_{\mathrm{r}}=1,5$ e degenerado para $\varepsilon_{\mathrm{r}}=1,05$. Parâmetros: $\mathrm{r}_{0}=63,17 \mathrm{~mm}, \mathrm{r}_{1}=0,8 \mathrm{r}_{0}$, $r_{2}=r_{0}+d, d=14 \mathrm{~mm}$. 